\title{
Influence des éclaircies sur les contraintes de croissance du hêtre
}

\author{
H. POLGE \\ I.N.R.A., Station de Recherches sur la Qualité des Bois \\ Centre de Recherches forestières de Nancy, \\ Champenoux, F 54280 Seichamps
}

\section{Résumé}

320 arbres ont été sondés, à raison de 2 prélèvements opposés par arbre, dans un dispositif en carré latin comportant 4 modalités de traitement : éclaircie ultra-forte, forte, faible et témoin. Le niveau des contraintes de croissance est apprécié à partir des diamètres axiaux et tangentiels, mesurés au micron près, de carottes de sondage de $5 \mathrm{~mm}$ de diamètre.

Sur les arbres droits, on observe un effet du traitement, significatif au seuil de 1 p. 100 , sur les 2 diamètres, le retrait radial et le rapport du retrait tangentiel au retrait radial; les diamètres et l'anisotropie du retrait augmentent régulièrement, et le retrait radial diminue au fur et à mesure que l'intensité des éclaircies augmente.

Sur les arbres penchés, les deux diamètres sont plus faibles, et les 3 retraits plus forts du côté tendu que du côté comprimé des tiges. Il n'y a pas d'effet significatif du traitement sur les diamètres du côté tendu, mais des effets très significatifs du côté opposé. D'une façon générale, la séparation des traitements est moins nette que sur les arbres droits, mais l'ordre de succession des intensités d'éclaircie est encore respecté.

Les résultats obtenus sur l'ensemble des arbres confirment les précédents, avec une discrimination encore plus nette, puisque, pour la somme des diamètres ixiaux et tangentiels par exemple, les 4 modalités de traitement se différencient parfaitement les unes des autres au seuil de 1 p. 100.

Des corrélations négatives apparaissent entre les diamètres des carottes et le retrait axial, confirmant l'association entre contraintes élevées et forte proportion de bois de tension. Sur les arbres penchés, les diamètres diminuent et le retrait axial augmente avec l'angle d'inclinaison des tiges.

L'expérience montre clairement que des éclaircies vigoureuses doivent être pratiquées si l'on veut réduire l'ampleur des contraintes de croissance dans les peuplements de hêtres.

\section{1. - Introduction}

Les contraintes de croissance des arbres forestiers, dont limportance économique est maintenant bien perçue dans de nombreux pays, ont fait l'objet au cours des 3 dernières décennies de nombreuses études et de 2 analyses bibliographiques successives : Dinwoodie (1966) et T.R.A.D.A. (1978).

En fait, tous les arbres sont le siège de contraintes de signe et de direction variés : contraintes longitudinales de traction à la périphérie du tronc et de compression dans 
la partie centrale, et contraintes transversales passant inversement de la compression à la traction dans le sens écorce-moelle (KuBLER, 1959).

Les plus graves sont les contraintes de tension axiale qui peuvent atteindre chez le hêtre 4000 newtons $/ \mathrm{cm}^{2}$, mais qui commencent à présenter des inconvénients technologiques sérieux dès lors qu'elles dépassent (c'est, semble-t-il, le cas pour environ 15 p. 100 des hêtres français) un seuil que lon peut provisoirement fixer aux alentours de 1500 newtons $/ \mathrm{cm}^{2}$.

Ces forces considérables se trouvent en effet libérées tout dabord lors de la réalisation du trait d'abattage et des découpes successives - elles peuvent à ce stade entraîner des fentes dans les billes concernées, voire même leur éclatement - puis lors du débit en scierie - elles risquent alors d'occasionner de nouvelles fissurations et aussi des déviations du trait de scie, d'où des changements d’épaisseur ou des défauts de planéité des sciages. Il en résulte au total des pertes de matières importantes, et parfois un déclassement des produits.

11 convient de noter que ces diverses manifestations des contraintes de croissance sont semblables à celles que provoque la rétractibilité du bois lors du séchage (la quasi-totalité des utilisateurs les confondent sous le nom de «nervosité » du bois); il s’agit cependant de deux phénomènes fondamentalement différents puisque la libération des contraintes intervient sur du bois frais, alors que le retrait ne commence à se manifester que lorsque l'humidité du bois est descendue au-dessous du point de saturation des fibres, c'est-à-dire au-dessous de 30 p. 100.

On peut observer également que les contraintes longitudinales périphériques, lorsqu'elles ne sont pas excessives, présentent un effet bénéfique : elles permettent (KUBLER, op. cit.) à un arbre soumis à un vent violent de résister aux forces de compression élevées qui s'exercent du côté fléchi et qui, sans cette «précontrainte» de tension naturelle, risqueraient de dépasser la limite de résistance des fibres qui est beaucoup plus faible dans cette direction que dans celle de la traction.

Quant à l'origine des contraintes de croissance, elle est encore fort controversée, mais doit être, au moins pour partie, recherchée (BoyD, 1972) dans l'épaississement et la lignification des cloisons cellulaires, lors du dépôt des couches successives de la membrane, qui entraînent une augmentation du diamètre radial des cellules et, comme conséquence de cette expansion transversale, par effet Poisson, leur contraction longitudinale.

La meilleure méthode de référence pour l'appréciation des contraintes sur des arbres vivants est, semble-t-il, celle qui consiste (Gueneau \& KIKATA, 1973) à coller une jauge d'extensométric à la surface du bois et à déterminer, à l'aide d'un pont de mesure, les microdéformations qu'elle subit quand on libère les forces qui s’exercent au point considéré, en creusant au-dessus et en dessous de la jauge 2 trous de $3 \mathrm{~cm}$ de diamètre et de $3 \mathrm{~cm}$ de profondeur. Il est cependant clair que ce procédé, au demeurant assez long à mettre en cuvre, est beaucoup trop traumatisant pour pouvoir être employé impunément sur un grand nombre d'arbres.

Une autre méthode, tout à fait différente, a été mise au point à la Station de Recherches sur la Qualité des Bois du C.N.R.F. (Polge \& Thiercel.n, 1979) : elle est basée sur la mesure au micron près des diamètres axiaux et tangentiels à l'état frais de simples carottes de sondage de $5 \mathrm{~mm}$, et part du principe que le petit cylindre de bois qu'elles représentent est retiré de l'arbre à l'état contraint et que la libé- 
ration, par leur prélèvement même, des forces qui s'exercent sur elles in situ doit entraîner des modifications de diamètre d'autant plus marquées que ces forces étaient plus élevées.

Cette hypothèse a été vérifiée lors d'une expérience menée en collaboration avec le Centre Technique du Bois de Paris dans laquelle ont été étudiées 430 carottes de sondage prélevées sur 86 arbres répartis dans les principales hêtraies françaises, et où diamètres tangentiels des carottes et microdéformations axiales mesurées par jauges d'extensométrie étaient liés de façon significative au seuil de 1 p. 1000 (Coefficients de corrélation entre moyennes d'arbres de $-0,68$ et intra-arbre de $-0,79$ ). En revanche, il n'y avait aucune liaison microdéformations axiales - diamètres axiaux, en raison vraisemblablement de la faible variabilité de ce diamètre par rapport à la précision du capteur utilisé à l'époque. Dans une expérience ultérieure en effet, qui fut entreprise après une amélioration sensible du système d'acquisition des mesures de diamètre, cette même corrélation s'est avérée, elle aussi, significative, mais au seuil de 1 p. $100(\mathrm{r}=-0,60$ avec 19 couples de données).

Le signe de la corrélation avec le diamètre tangentiel est très surprenant, puisqu'on libère dans cette direction des contraintes de compression, ct aussi parce que la contraction dans le sens du fil du bois que provoque la suppression des efforts de traction axiale devrait entrainer, par effet Poisson, une dilatation tangentielle d'autant plus élevée que la contrainte elle-même était plus forte. De difficiles recherches seront nécessaires pour rendre totalement compte de l'ensemble du phénomène, et ce d'autant que, lors d'une expérience de simulation réalisée sur des échantillons parallélipipédiques frais de hêtre soumis sur une machine d'essais à des efforts connus, soit de traction longitudinale, soit de compression tangentielle, Ferrand (1979) a montré au contraire que le diamètre tangentiel des carottes prélevées sous contrainte augmentait de façon très significative avec la charge appliquée dans l'une ou lautre direction.

Cette contradiction apparente est sans doute due au fait que, chez le hêtre, contraintes élevées et forte proportion de bois de tension sont souvent associées (LENZ \& Strassler, 1959, Trenard \& Gueneau, 1975) et les particularités de ce type de bois de réaction, qui diffère du bois normal au point de vue anatomique (membranes plus épaisses, angles des microfibrilles plus faibles), rhéologique (module d'élasticité plus élevé) et chimique (teneur en lignine réduite) pourraient (BoyD, commmunication personnelle) fournir un début d'explication.

Quoi qu’il en soit, pour la pratique courante, le résultat essentiel peut être considéré comme acquis : les arbres ayant de fortes contraintes de traction axiale (qui se manifestent sous forme de microdéformations élevées dans la méthode de mesure par jauges d'extensométrie) donnent des carottes de sondage ayant des diamètres, et en particulier un diamètre tangentiel, faibles. On peut ainsi, à condition d'étudier un nombre d'arbres suffisant, comparer, de façon non destructive et non traumatisante pour l'arbre, l'état de contrainte de diverses populations de hêtre pour connaître l'effet des diverses sources biologiques de variation possibles (génotype, milieu, traitement) sur cette importante caractéristique technologique.

L'expérience dont il est rendu compte ci-dessous concerne un de ces facteurs : l'intensité des éclaircies, dont, sauf erreur, l'influence sur les contraintes de croissance n'avait jamais été étudiée jusqu'ici. 


\section{2. - Matériel et méthode}

\section{1. - Dispositif expérimental}

L'étude a été entreprise dans le dispositif en carré latin $4 \times 4$ installé par la Station de Sylviculture et de Production du C.N.R.F. en Forêt Domaniale de Souilly (Meuse) (*).

L'expérience, implantée en 1963 dans un perchis régulier de hêtres alors âgés en moyenne d'environ 35 ans comporte 4 modalités (Station de Sylviculture et de Production, 1970) :

- Eclaircie Ultra Forte : « le but est d'obtenir le plus rapidement possible des arbres de gros diamètre de qualité déroulage ", en s'inspirant d'une table de production danoise de C.M. Moller et en visant à avoir, en fin de révolution à 120 ans, 116 tiges à l'hectare.

- Eclaircie Forte : norme déduite de la table de production Schober en $1^{\text {r" }}$ classe de fertilité; objectif : 160 tiges à l'ha à 120 ans.

- Eclaircie faible : correspond à un type de sylviculture très prudente autrefois pratiquée dans les hêtraies françaises et traduit en chiffres par la table de production PARDE pour la Forêt de Haye; 240 tiges à l'hectare prévues à 120 ans.

- Témoin : seuls sont enlevés les arbres morts et dépérissants.

Trois passages en éclaircie ont été réalisés en 1963, 1969 et 1975 ; immédiatement après ce dernier, les volumes moyens inventoriés étaient les suivants (OswaL.D, communication personnelle) :

- Eclaircie Ultra Forte : $185 \mathrm{~m}^{3 / \text { ha }}$ pour 566 tiges.

- Eclaircie Forte : $225 \mathrm{~m}^{3}$ pour 936 tiges.

- Eclaircie faible : $251 \mathrm{~m}^{3}$ pour 1282 tiges.

- Témoin : $302 \mathrm{~m}^{3}$ pour 1510 tiges.

\section{2. - Echantillonnage}

20 arbres ont été sondés par parcelle unitaire, à raison de 2 sondages diamétralement opposés par individu, soit $20 \times 2 \times 4 \times 4=640$ échantillons.

Pour éviter d'éventuelles erreurs dans les calculs d'accroissement susceptibles d'être occasionnées par le léger traumatisme causé par les sondages, le plus grand nombre possible d'arbres (248) ont été pris dans les zones séparatives entre placeaux, dont chaque moitié a subi le même traitement que la parcelle unitaire limitrophe; ainsi, seules 72 tiges ont dû être sondées dans le dispositif proprement dit.

(*) Il s'agit d'un carré latin de $4 \times 4=16$ placettes d'une superficie de 20 ares chacune, séparées et entourées de bandes de protection de 10 mètres de large. 
De même, tenant compte de l'effet possible de l'inclinaison du tronc sur le développement du bois de tension et sur le niveau des contraintes, on s'est efforcé d'éliminer de l'échantillonnage les arbres penchés; malheureusement, il n'a été possible de recruter que 173 individus parfaitement verticaux qui ont alors été sondés suivant une direction aléatoire. La pente des 147 autres a été mesuréc de façon précise sur les 2 mètres inférieurs de la tige, et les sondages ont été exécutés suivant la direction de l'inclinaison, précisément afin de pouvoir comparer les niveaux de contrainte entre le côté tendu et le côté opposé considéré comme «normal». En raison de l'hétérogénéité du peuplement, la proportion d'arbres droits varie d'un placeau à l'autre (de 35 p. 100 à 75 p. 100), mais une certaine homogénéisation se produit au niveau des traitements (étendue de variation limitée de 54 p. 100 à 60 p. 100$)$.

\section{3. - Prélèvements et mesures}

Une première expérience entreprise dans le même dispositif en 1977 et pour laquelle plusieurs tarières de sondages différentes avaient été utilisées avait montré qu'il existait un effet propre de l'outil sur les diamètres axiaux et tangentiels à l'état frais des carottes obtenues, effet qui est dû vraisemblablement atıx différences de géométrie et d'élasticité du biseau d'un instrument à l'autre (Polcil, 1979).

Tenant compte de cet enseignement, tous les prélèvements nécessaires pour cette nouvelle étude ont été faits avec la même tarière sans réaffutage (tarière bois dur «finland» de $5 \mathrm{~mm}$ de diamètre). Un guide a été utilisé (REINEkı, 1941) pour éviter toute ondulation des carottes dans la zone périphérique qui aurait pu perturber les mesures de diamètre. Les échantillons ont été placés dans l'eau aussitôt après extraction et passés au capteur dans les jours suivants. Les valeurs de diamètre retenues sont les moyennes de 80 mesures faites au pas de $0,25 \mathrm{~mm}$ entre $5 \mathrm{~mm}$ et $25 \mathrm{~mm}$ à partir de l'écorce. La même procédure a été retenue pour les mesures à l'état dit «sec à l'air » destinées à l'étude des rétractibilités axiales et tangentielles, après conditionnement des échantillons à 10 p. 100 d'humidité dans une étuve hygrométrique. Les déterminations de longueur pour le calcul du retrait radial ont été faites avec un micromètre au $1 / 100^{\circ}$ de $\mathrm{mm}$ après section des 2 extrémités suivant des plans rigoureusement perpendiculaires à l'axe à l"aide d'une scie circulaire miniature et positionnement des carottes dans une gouttière en forme de $\mathrm{V}$.

Les infra-densités ont été obtenues en fin de chaîne par la méthode de saturation intégrale déjà décrite (Pol.gE, 1963).

\section{3. - Résultats}

Les analyses ont porté sur 8 variables : les diamètres axial et tangentiel des carottes à l'état frais, ainsi que leur somme, qui rendent compte de l'état de contrainte des arbres, les retraits axial, tangentiel et radial, le rapport des deux derniers, qui caractérise l'anisotropie du retrait, et l'infra-densité. 
Elles ont été faites

- d'une part séparément sur les arbres droits et sur les arbres penchés, après dans les deux cas, ajustement des données pour tenir compte de la non-orthogonalité du dispositif ;

- d'autre part globalement sur l'ensemble des arbres (dispositif orthogonal) en prenant pour les arbres droits la moyenne des valeurs obtenues pour les deux échantillons opposés, et, pour les arbres penchés, celle de la seule carotte prélevée du côté comprimé, considérée comme normale.

\section{1. - Etude des arbres droits}

Les résultats de l'analyse de variance sont indiqués dans le tableau ci-dessous qui donne, pour les différentes sources de variation, les valeurs du test $F$ F de Snédécor, ainsi que leur degré de signification (** pour le seuil de 1 p. 100 , * pour celui de 5 p. 100, et NS pour non significatif), compte tenu des degrés de liberté qui sont de 3 et 335 pour les effets ligne, colonne et traitement, et de 1 et 335 pour leffet position dans l'arbre (2 prélèvements opposés).

\section{Tableau 1}

Analyse de variance des arbres droits

Variance analysis of straight stems

\begin{tabular}{|c|c|c|c|c|}
\hline & \multicolumn{4}{|c|}{ Source de variation } \\
\hline & Ligne & Colonne & Traitement & Position \\
\hline$\varnothing$ axial & $48,90 * *$ & $26,26 * \cdots$ & $8,00 * *$ & $0,27 \mathrm{NS}$ \\
\hline$\ldots \ldots \ldots \ldots$ & $18,50 * *$ & $14,90 *$ & $28,01: *$ & $6,14 *$ \\
\hline$\varnothing$ axial + tangentiel & $38,91 * *$ & $25,44 *$ & $28,17 * *$ & $4,67 *$ \\
\hline Retrait axial & $0,66 \mathrm{NS}$ & $2,39 \mathrm{NS}$ & $1,12 \mathrm{NS}$ & $0,82 \mathrm{NS}$ \\
\hline Retrait tangentiel & $2,58 \mathrm{NS}$ & $6,59 * *$ & $1,98 \mathrm{NS}$ & $0,11 \mathrm{NS}$ \\
\hline Retrait radial ... & $3,66 *$ & $1,99 \mathrm{NS}$ & $10,31 * *$ & $0.00 \mathrm{NS}$ \\
\hline Anisotropie retrait & $0,82 \mathrm{NS}$ & $2,63 *$ & $13,00 *$ & $0,00 \mathrm{NS}$ \\
\hline Infradensité & $2,90 *$ & $2,14 \mathrm{NS}$ & $0,80 \mathrm{NS}$ & $1,27 \mathrm{NS}$ \\
\hline
\end{tabular}

Source de variation : source of variation.

Ligne : line.

Colonne : column.

Traitement : treatment.

Position : position.

Diamètre : diameter.

Axial : axial.

Tangentiel : tangential.

Retrait : Shrinkage.

Infradensité : specific gravity.

Les effets ligne et colonne sont très marqués pour la plupart des caractéristiques mesurées, et, en particulier pour les diamètres des carottes à l'état frais ; ceci justifie 
a posteriori l'utilité du dispositif en carré latin, et montre en outre que les critères retenus, notamment ceux qui sont liés aux contraintes de croissance sont très sensibles à l'action des facteurs du milieu. Toutes les variables augmentent ou diminuent de façon progressive, et dans l'ordre des lignes, de la première à la dernière; ceci paraît traduire un effet de gradient qui ne surprend guère puisque la pente prépondérante du terrain a une direction perpendiculaire à celle des lignes. Les variations entre colonnes ne suivent jamais l'ordre de succession sur le terrain, et aucune explication ne peut être proposée à leur sujet.

L'effet position est très peu marqué ; il n'aurait même jamais dû être significatif, puisqu'il s'agit ici théoriquement d'arbres droits sur lesquels on a procédé à deux prélèvements opposés de direction aléatoire; la seule influence significative s'exerce (au seuil de 5 p. 100 seulement) sur le diamètre tangentiel, qui est le plus sensible au traitement et, on le verra plus loin, à l'inclinaison des tiges (l'effet sur la somme des diamètres n'est manifestement qu'une conséquence du précédent); on peut penser que les sondages n'ont pas été faits aussi au hasard qu'on le souhaitait, et qu'une majorité d'entre eux ont été exécutés par malchance dans une direction privilégiée par rapport à la concurrence des voisins ou à une inclinaison qui aurait pu échapper aux opérateurs.

Les tests de comparaison de moyennes sur la moyenne des deux positions donnent les résultats suivants (les abréviations UF, $\mathrm{F}$, f et $\mathrm{t}$ correspondent dans l'ordre aux traitements d'éclaircies ultra-fortes, fortes, faibles et aux témoins) :

\begin{tabular}{|c|c|c|c|c|}
\hline$\varnothing$ axial en microns $\ldots \ldots$ & $\begin{array}{r}\mathrm{t} 4990,9 \\
\end{array}$ & f 4992,7 & F 4995,1 & UF 4995,3 \\
\hline$\varnothing$ tangentiel & $\begin{array}{r}\mathrm{t} 4974,7 \\
\end{array}$ & f. 4981,1 & F 4988,5 & UF 4991,5 \\
\hline$\varnothing$ axial + tangentiel & $\begin{array}{r}t 9965,6 \\
\end{array}$ & f 9973,8 & F 9983,6 & UF 9986,8 \\
\hline Retrait radial $(\%)$ & UF 3,5265 & F 3,5270 & f 3.7720 & t 3.8112 \\
\hline Anisotropie retrait & $1 \quad \mathrm{t} 3,070$ & f 3,108 & F 3,368 & UF 3,441 \\
\hline
\end{tabular}

Les valeurs situées dans des accolades séparées étant différentes les unes des autres au seuil de 1 p. 100 , l'effet remarquable du traitement sur ces divers critères de qualité du bois apparaît clairement puisque les modalités d'éclaircie se succèdent exactement dans l'ordre de leur intensité, et que les seuls regroupements intéressent des traitements voisins : témoins et éclaircie faible, ou éclaircie forte et ultra-forte.

On peut noter également que les diamètres axiaux sont toujours supérieurs aux tangentiels, et que l'écart entre eux varie, lui aussi en fonction du traitement, puisqu'il diminue régulièrement de 16,2 microns pour les témoins à 3,8 pour l'éclaircie ultraforte, en passant par 11,6 pour la modalité faible et 6,6 pour la forte. 


\section{2. - Êtude des arbres penchés}

Une première analyse de variance, prenant en compte les 4 sources de variation retenues pour les arbres droits a confirmé l'effet très important du facteur «position». Le tableau ci-après donne pour toutes les variables pour lesquelles cet effet est significatif la valeur du test $\ll F »$, ainsi que les moyennes pour les deux positions opposées :

\section{Tableau 2}

Comparaison des prélèvements côté tendu et côté comprimé sur les arbres penchés Comparison of upper and lower cores on leaning stems

\begin{tabular}{|c|c|c|c|}
\hline & \multirow{2}{*}{ « F» } & \multicolumn{2}{|c|}{ Position } \\
\hline & & Comprimée & Tendue \\
\hline Diamètre axial (en microns) & $5,12 *$ & 4990,1 & 4988,6 \\
\hline Diamètre tangentiel (en microns) & $210,16 * *$ & 4987,9 & 4954,8 \\
\hline Diamòtre axial + diamètre tangentiel & $186,65 * *$ & 9978,1 & 9943,5 \\
\hline Retrait axial (en $\%) \ldots \ldots \ldots \ldots$ & $196,43 * *$ & $-0,150$ & $+0,331$ \\
\hline Retrait tangentiel (en $\%$ ) & $55,70 * * *$ & 11,865 & 12,690 \\
\hline Retrait radial (en \%) & $5,77 *$ & 3,527 & 3,667 \\
\hline
\end{tabular}

Position comprimée : lower sample.

Position tendue : upper sample.

Les deux diamètres sont plus faibles et les trois retraits plus élevés sur la face supérieure que sur la face inférieure des arbres penchés; cela signifie que les contraintes y sont plus fortes et le bois de tension plus abondant, ce qui ne saurait surprendre, mais oblige à étudier séparément les échantillons côté tendu (référence 《 $T$ ») et comprimé ( $C$ 》). Le tableau ci-après donne les résultats comparés des analyses de variance les concernant sous l'effet des trois autres sources de variation :

\section{TABLEAU 3}

Analyse de variance des arbres penchés Variance analysis of leaning stems

\begin{tabular}{|c|c|c|c|c|c|c|}
\hline & \multicolumn{2}{|c|}{ Effet ligne } & \multicolumn{2}{|c|}{ Effet colonne } & \multicolumn{2}{|c|}{ Effet traitement } \\
\hline & $\mathrm{T}$ & $\mathrm{C}$ & $\mathrm{T}$ & $\mathrm{C}$ & $\mathrm{T}$ & $\mathrm{C}$ \\
\hline Diamètre axial & $22,54 * 3$ & $38,96 \div$ & $8,76 * *$ & $10,30 * *$ & $2,46 \mathrm{NS}$ & $4,11 * *$ \\
\hline Diamètre tangentiel & $2,26 \mathrm{NS}$ & $8,65 * *$ & $0,37 \mathrm{NS}$ & $11,07 * *$ & $1,58 \mathrm{NS}$ & $17,50 * *$ \\
\hline Diamètres axial + tangentiel & $5,02 * *$ & $31,19 * *$ & $0,92 \mathrm{NS}$ & $19,69 *$ & 1,89 NS & $21,38 * *$ \\
\hline Retrait axial & $0,74 \mathrm{NS}$ & $3,94 * *$ & $1,40 \mathrm{NS}$ & $3,00 *$ & $2,44 \mathrm{NS}$ & $2,13 \mathrm{NS}$ \\
\hline Retrait tangentiel & $1,43 \mathrm{NS}$ & $1,41 \mathrm{NS}$ & 1,84. NS & $4,43 * *$ & $11,32 * *$ & $4,49 * *$ \\
\hline Retrait radial & $1,79 \mathrm{NS}$ & $1,32 \mathrm{NS}$ & $0,35 \mathrm{NS}$ & $0,25 \mathrm{NS}$ & $5,95 * *$ & $4,09 * *$ \\
\hline Anisotropie retrait $(\mathrm{Rt} / \mathrm{Rr})$ & $0,84 \mathrm{NS}$ & $1,16 \mathrm{NS}$ & $1,92 \mathrm{NS}$ & $2,06 \mathrm{NS}$ & $10,56 * *$ & $7,81 * *$ \\
\hline Infra-densité & $1,66 \mathrm{NS}$ & $1,82 \mathrm{NS}$ & $2,11 \mathrm{NS}$ & $2.01 \mathrm{NS}$ & $1,24 \mathrm{NS}$ & $2,03 \mathrm{NS}$ \\
\hline
\end{tabular}

Effet ligne : effect of the line.

Effet colonne : effect of the column.

Effet traitement : effect of the treatment. 
Les effets ligne et colonne sont nettement moins marqués que sur les arbres droits, notamment en ce qui concerne le côté tendu qui apparaît ainsi comme relativement peu soumis à l'action du milieu.

Les résultats des tests de comparaison de moyennes pour l'effet traitement sont indiqués ci-dessous au seuil de probabilité de 1 p. 100 (lorsqu'il existe une influence significative sur une seule des deux positions, les valeurs moyennes sont également données pour l'autre, dans un ordre croissant, à titre d'information) :

\section{Position}

\begin{tabular}{|c|c|c|c|c|c|c|c|c|c|}
\hline \multirow{2}{*}{$\varnothing$ axial $\ldots \ldots \ldots \ldots$} & $\mathrm{C}$ & & 4987,6 & & 4990,0 & & 4990.9 & UF & 4991,9 \\
\hline & $\mathrm{T}$ & & 4987,3 & & 4987.6 & & 4988.7 & $\mathrm{UF}$ & 4990,9 \\
\hline \multirow[t]{2}{*}{$\varnothing$ tangentiel } & C & & 4979,3 & & 4987,5 & & $4,988,2$ & $\mathrm{UF}$ & 4996,2 \\
\hline & $\mathbf{T}$ & & 4949,9 & & 4951,0 & & 4956,7 & UF & 4961,9 \\
\hline \multirow[t]{2}{*}{$\varnothing$ axial + tangentiel } & $\mathrm{C}$ & & 9966,9 & & 9977,5 & & 9979,1 & UF & 9988,0 \\
\hline & $\mathbf{T}$ & & 9937,5 & & 9939,7 & & 9943,9 & UF & 9952,8 \\
\hline \multirow[t]{2}{*}{ Retrait tangentiel } & $\mathrm{C}$ & & 11,430 & & 11.719 & & 12,110 & UF & 12,152 \\
\hline & $T$ & & 12,016 & f & 12,427 & $\mathbf{F}$ & 13,035 & UF & $\begin{array}{r}13,213 \\
\end{array}$ \\
\hline \multirow[t]{2}{*}{ Retrait radial $\ldots \ldots \ldots \ldots$} & $\mathrm{C}$ & UF & 3,300 & $\mathrm{~F}$ & 3,512 & $\mathrm{f}$ & 3,645 & $t$ & 3,659 \\
\hline & $\mathbf{T}$ & $\begin{array}{l}\text { UF } \\
\end{array}$ & $\begin{array}{r}3,353 \\
-\end{array}$ & F & 3,699 & $\mathrm{f}$ & 3,798 & $\mathrm{t}$ & 3,823 \\
\hline \multirow[t]{2}{*}{ Anisotropie retrait $\ldots \ldots$} & $\mathrm{C}$ & $\mathrm{T}$ & 3,220 & $\mathrm{f}$ & 3,249 & $\mathrm{~F}$ & $\begin{array}{l}3,472 \\
\end{array}$ & UF & 3,763 \\
\hline & $\mathrm{T}$ & T & 3,196 & $\mathrm{f}$ & $\begin{array}{r}3,319 \\
\\
\end{array}$ & $F$ & 3,613 & $\begin{array}{c}\text { UF } \\
\vdots\end{array}$ & 4,100 \\
\hline
\end{tabular}

On constate tout d'abord que la différenciation des traitements se fait moins bien que sur les arbres droits ; cela est déjà vrai pour les échantillons côté comprimé, où souvent trois modalités d'éclaircie sont regroupées dans une même accolade, mais encore plus pour le côté tendu, où aucune différence significative n'existe sur les diamètres des carottes de sondage. Mais, comme pour les individus verticaux, on ne relève aucune «anomalie», c'est-à-dire que l'on ne retrouve jamais un traitement groupé avec un autre très différent du point de vue sylvicole, alors qu'un plus voisin s'en séparerait. Fréquemment les diverses modalités s'échelonnent dans l'ordre des intensités d'éclaircie, et la plus sévère notamment se différencie à plusieurs reprises de toutes les autres.

La faible valeur des diamètres tangentiels des prélèvements du côté tendu doit être soulignée : elle confirme l'ampleur des contraintes de croissance qui s'y exercent. 
Enfin, il existe une règle à peu près générale qui veut que les diamètres tangentiels des carottes à l'état frais soient inférieurs aux diamètres axiaux; cela est vrai, on l'a vu, pour les arbres droits; c'est encore vérifié pour les prélèvements faits sur la face tendue des arbres penchés, mais plus pour la face comprimée où la différence entre diamètre axial et tangentiel, après avoir diminué de 8,3 microns chez les témoins à 2,5 et 2,7 chez les individus soumis à des éclaircies faible et forte, devient négative ( $-4,3$ microns) pour la modalité ultra-forte. Aucune explication ne peut être proposée pour rendre compte de cette particularité qui caractérise le côté le moins contraint des arbres bénéficiant du traitement engendrant les plus faibles contraintes; mais le fait méritait d’être signalé, car un test de comparaison de moyennes montre que la supériorité du diamètre tangentiel sur le diamètre axial est, pour ces échantillons, significative au seuil de 1 p. 100.

\section{3. - Etude de licnsemble des arbres}

L'analyse de variance portant sur les prélèvements côté comprimé des arbres penchés et sur la moyenne des deux sondages pour les arbres droits donne les résultats suivants:

TABLEAU 4

Analyse de variance pour l'ensemble des arbres Variance analysis for all the stems

\begin{tabular}{|c|c|c|c|}
\hline & $\begin{array}{l}\text { Effet } \\
\text { ligne }\end{array}$ & $\begin{array}{c}\text { Effet } \\
\text { colonne }\end{array}$ & $\begin{array}{c}\text { Effet } \\
\text { traitement }\end{array}$ \\
\hline Diamètre axial $\ldots \ldots \ldots \ldots \ldots \ldots \ldots$ & $52,70 * *$ & $24,73 * *$ & $8,61 *: 5$ \\
\hline Diamètre tangentiel & $21,55 *$ & $22,97 * *$ & $38,22 * *$ \\
\hline Diamètre axial + tangentiel $\ldots$ & $49,14: *$ & $36,24 *:$ & $38,50 * *$ \\
\hline Retrait axial ..... & $0,89 \mathrm{NS}$ & $1,07 \mathrm{NS}$ & $1,59 \mathrm{NS}$ \\
\hline Retrait tangentiel $\ldots \ldots \ldots \ldots \ldots \ldots$ & $1,96 \mathrm{NS}$ & 8,87 ** & $5,98 * *$ \\
\hline Retrait radial $\ldots \ldots \ldots \ldots \ldots$ & $3,57 *$ & $0,82 \mathrm{NS}$ & $10,22 * *$ \\
\hline Anisotropie du retrait .. & $1,35 \mathrm{NS}$ & $3,47 *$ & $17,52 * *$ \\
\hline Infra-densité $\ldots \ldots \ldots \ldots$ & $2,13 \mathrm{NS}$ & $1,70 \mathrm{NS}$ & $1,74 \mathrm{NS}$ \\
\hline
\end{tabular}

Les résultats des tests de comparaison de moyennes correspondants sont les suivants (comme précédemment, et compte tenu de l'ampleur des différences, seul le scuil de probabilité de 1 p. 100 a été retenu) : 




Ces résultats confirment ceux obtenus sur les seuls arbres droits, mais avec une discrimination encore plus nette, puisque, non seulement les traitements se suivent dans lordre des intensités d"éclaircie, mais encore, pour trois variables, dont deux particulièrement importantes pour l'appréciation des contraintes (le diamètre tangentiel et la somme des deux diamètres), les 4 modalités de traitement se distinguent très significativement les unes des autres.

Il était dès lors tentant de voir dans quelle mesure ces diamètres pouvaient être liés aux divers paramètres permettant de quantifier l'intensité des éclaircies; la meilleure liaison mise en évidence est celle qui existe entre le diamètre tangentiel moyen des carottes par traitement et la surface terrière correspondante: le coefficient de corrélation est de --0,995, significatif au seuil de 1 p. 100 (figure 1).

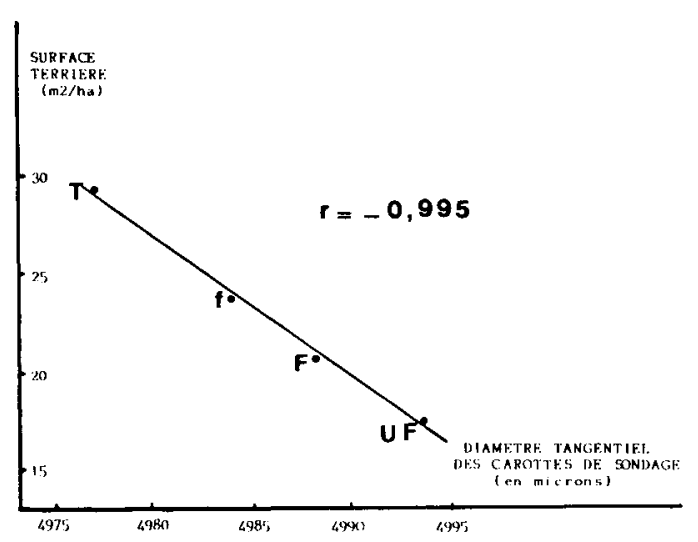

FIG. 1

Relation entre la surface terrière et le diamètre tangentiel des carottes de sondage Relationship between basal area and tangential diameter of increment core 
Quelques observations intéressantes peuvent être faites au sujet de la variabilité des divers caractères; le tableau ci-dessous donne les écarts-types moyens des diamètres de carottes et des retraits, dans les deux directions et par traitement, pour la moyenne des deux prélèvements des arbres droits d'une part (référence « $D$ »), et pour les prélèvements côté comprimé $(C)$ et côté tendu (T) des arbres penchés d'autre part :

\section{Tableau 5}

Ecarts types comparés des prélèvements sur arbres droits et sur les côtés tendus et comprimés des arbres penchés

Comparative standard errors for straight stem samples and for upper and lower samples of the leaning stems

\begin{tabular}{|c|c|c|c|c|c|c|c|}
\hline & \multirow{2}{*}{$\begin{array}{l}\text { Traite- } \\
\text { ment }\end{array}$} & \multicolumn{3}{|c|}{ Sens axial } & \multicolumn{3}{|c|}{ Sens tangentiel } \\
\hline & & $\mathrm{D}$ & $\mathrm{C}$ & $\mathrm{T}$ & $\mathrm{D}$ & $\mathrm{C}$ & $\mathrm{T}$ \\
\hline \multirow[t]{4}{*}{ Diamètres en microns } & UF & 6,86 & 4,16 & 5,57 & 14,12 & 8,16 & 25,94 \\
\hline & $F$ & 7,44 & 5,80 & 7,75 & 12,29 & 10,28 & 29,17 \\
\hline & f & 8,23 & 5,87 & 6,75 & 11,80 & 8,25 & 20,82 \\
\hline & $t$ & 5,88 & 5,01 & 5,29 & 12,10 & 8,28 & 25,16 \\
\hline \multirow[t]{4}{*}{ Retraits en $\%$} & UF & 0.17 & 0,09 & 0.40 & 1,14 & 1,15 & $1,0,3$ \\
\hline & $\mathrm{F}$ & 0,14 & 0,09 & 0,41 & 1,06 & 0,81 & 0,94 \\
\hline & $\mathrm{f}$ & 0,15 & 0,12 & 0,28 & 0,77 & 0,69 & 0,66 \\
\hline & $\mathrm{t}$ & 0,18 & 0,05 & 0,34 & 0.76 & 0,83 & 0,67 \\
\hline
\end{tabular}

Sens axial : axial direction.

Sens tangentiel : tangential direction.

L'effet du traitement sur la variabilité est faible puisquine seule tendance nette se manifeste : elle concerne le retrait tangentiel où, quels que soient les prélèvements, l'écart-type est sensiblement plus élevé pour les éclaircies forte et ultra-forte que pour la faible et le témoin : cet écart va dans le même sens que celui qui affecte les retraits eux-mêmes, mais son ampleur est bien supérieure, conduisant à des coefficients de variation nettement différents.

L'effet de la position de prélèvement est, en revanche très marqué : si l’on excepte le retrait tangentiel en effet, la variabilité des échantillons «comprimés» est toujours la plus faible (souvent de beaucoup), et celle des échantillons «tendus» est de loin la plus forte pour le retrait axial et pour les diamètres tangentiels; ces observations se vérifiant en général au niveau de chaque parcelle unitaire, le phénomène apparaît donc comme indépendant à la fois du traitement et du milieur; la variabilité supérieure des échantillons «tendus» reflète sans doute celle des angles d'inclinaison; mais on doit noter que celle-ci est sans effet sur les caractéristiques mesurées côté comprimé, qui apparaissent au contraire comme relativement constantes, et tout se passe comme si les potentialités génétiques ne pouvaient s'y exprimer dans toute leur ampleur. On doit retenir en outre que si le bois «comprimé 》 est voisin du bois normal (celui des arbres droits) pour la valeur moyenne des critères de qualité du bois étudiés ici, il s’en distingue nettement quant à leur variabilité. 


\section{4. - Liaisons entre variables}

- Cas des arbres droits:

La matrice des coefficients de corrélation entre les divers critères mesurés est donnée ci-dessous, avec, dans chaque cas, à la ligne supérieure les cofficients entre moyennes de traitements, et à la ligne inférieure les coefficients «intra pour l'ensemble des individus de l'expérience:

\section{TABleau 6}

Coefficients de corrélation inter (lignes supérieures) et intra (lignes inférieures)

Between treatments (upper line) and within treatments (lower line) correlation coefficients

\begin{tabular}{|c|c|c|c|c|c|c|c|}
\hline $\begin{array}{l}\text { Diamètre } \\
\text { tangenticl }\end{array}$ & $\begin{array}{l}0,992^{* * *} \\
0,335^{* * *}\end{array}$ & & & & & & \\
\hline $\begin{array}{c}\text { Somne } \\
\text { des diamètres }\end{array}$ & $\begin{array}{l}0,995^{* * ;} \\
0,672^{* * *}\end{array}$ & $\begin{array}{l}1,000^{* * *} \\
0,923^{* * *}\end{array}$ & & & & & \\
\hline Retrait axial & $\begin{array}{l}\text { NS } \\
\text { NS }\end{array}$ & $\begin{array}{c}\text { NS } \\
-0260^{* *}\end{array}$ & $\begin{array}{c}\mathrm{NS} \\
-0,177^{* * *}\end{array}$ & & & & \\
\hline $\begin{array}{l}\text { Retrait } \\
\text { tangenticl }\end{array}$ & $\begin{array}{l}\text { NS } \\
\text { NS }\end{array}$ & $\begin{array}{l}0,957^{*} \\
\text { NS }\end{array}$ & $\begin{array}{l}0,952^{*} \\
\text { NS }\end{array}$ & $\begin{array}{l}0,998^{* * *} \\
0,152 * *\end{array}$ & & & \\
\hline Retrait radial & $\begin{array}{c}-0,971^{*} \\
\text { NS }\end{array}$ & $\begin{array}{l}-0,956^{*} \\
-0,215^{* *}\end{array}$ & $\mid \begin{array}{l}-0,960^{*} \\
-0,201^{* * *}\end{array}$ & $\begin{array}{l}\text { NS } \\
\text { NS }\end{array}$ & $\begin{array}{c}\text { NS } \\
0,154 * *\end{array}$ & & \\
\hline $\begin{array}{l}\text { Anisotropic } \\
\text { retrait }\end{array}$ & $\begin{array}{l}0,960^{*} \\
\text { NS }\end{array}$ & $\begin{array}{l}0,964^{*} \\
0,161^{* * ;}\end{array}$ & $\begin{array}{l}0,964^{*} \\
0,140^{* *}\end{array}$ & $\begin{array}{c}0,970^{*} \\
\text { NS }\end{array}$ & $\begin{array}{l}0,983^{*} \\
0,469 * *\end{array}$ & $\begin{array}{l}-0,988^{*} \\
-0,766^{* *}\end{array}$ & \\
\hline \multirow[t]{2}{*}{ Infra-densité } & $\begin{array}{c}\text { NS } \\
-0,342^{*}\end{array}$ & $\begin{array}{l}\text { NS } \\
\text { NS }\end{array}$ & \begin{tabular}{|c|}
$\mathrm{NS}$ \\
$-0,174^{* * 1}$
\end{tabular} & $\begin{array}{l}\text { NS } \\
\text { NS }\end{array}$ & $\begin{array}{c}\mathrm{NS} \\
0,175^{* *}\end{array}$ & $\begin{array}{c}\text { NS } \\
0,237 * *\end{array}$ & $\begin{array}{c}\text { NS } \\
-0,108 *\end{array}$ \\
\hline & $\varnothing$ & $\begin{array}{c}\varnothing \\
\text { tangent. }\end{array}$ & $\begin{array}{l}\text { Somme } \\
\text { des } \varnothing\end{array}$ & $\begin{array}{c}\text { Retrait } \\
\text { axial }\end{array}$ & $\begin{array}{c}\text { Retrait } \\
\text { tangent. }\end{array}$ & $\begin{array}{l}\text { Retrait } \\
\text { radial }\end{array}$ & $\begin{array}{l}\text { Retraits } \\
\text { tang./rad. }\end{array}$ \\
\hline
\end{tabular}

Somme des diamètres : sum of the diameters.

On observe tout d'abord qu’il n'y a aucun cas de corrélations « inter » et «intra » de signes contraires. Au reste, mis à part un certain nombre de liaisons dues en partie au mode de calcul (entre les deux diamètres et leur somme, ou encore entre l'anisotropie du retrait et ses composantes), on doit surtout noter :

- les corrélations intra négatives entre le retrait axial d'une part, et le diamètre tangentiel ou la somme des diamètres d'autre part, qui confirme les relations entre contraintes et bois de tension; 
- les liaisons intra positives entre l'infra-densité et les deux retraits transversaux, qui semblent bien correspondre à un phénomène général, et s'expliquent sans doute par le fait que ces 3 caractéristiques angmentent avec la proportion relative de membranes dans le plan ligneux;

- les corrélations négatives des diamètres avec le retrait radial, qui, combinées avec une tendance inverse avec le retrait tangentiel, conduisent aux liaisons positives défavorables observées avec l'anisotropie du retrait (à noter que l'ensemble des corrélations inter pouvaient se prévoir à partir des tests de comparaison de moyennes où les diamètres, le retrait tangentiel et lanisotropie variaient exactement dans le même sens, et le retrait radial, dans le sens contraire) ;

- l'absence totale de corrélations inter pour l'infra-densité, corrélations qui, en toute rigueur n'auraient même pas dû être calculées, puisque le « $F$ » traitement n'était pas significatif pour cette variable.

\section{- Cas des arbres penchés:}

Les seules corrélations retenues ici sont celles qui concernent linclinaison des tiges; les coefficients qui la lient aux divers critères de qualité sont les suivants (pour l'ensemble des individus penchés) :

\section{TABLEAU 7}

Corrélations entre l'angle d'inclinaison des arbres penchés et les diverses caractéristiques du bois

Correlation coefficients between the vertical angle of leaning stems and wood characteristics

\begin{tabular}{c|c|c|c|c|c|c|c|c}
\hline & $\begin{array}{c}\text { Diamètre } \\
\text { axial }\end{array}$ & $\begin{array}{c}\text { Diamètre } \\
\text { tangent. }\end{array}$ & $\begin{array}{c}\text { Somme } \\
\text { diamètre }\end{array}$ & $\begin{array}{c}\text { Retrait } \\
\text { axial }\end{array}$ & $\begin{array}{c}\text { Retrait } \\
\text { tangent. }\end{array}$ & $\begin{array}{c}\text { Retrait } \\
\text { radial }\end{array}$ & $\begin{array}{c}\text { Aniso- } \\
\text { tropie }\end{array}$ & $\begin{array}{c}\text { Infra- } \\
\text { densité }\end{array}$ \\
\hline $\begin{array}{c}\text { Côté compri- } \\
\text { mé } \ldots . . .\end{array}$ & $-0,188^{*}$ & $-0,320^{* *}$ & $-0,320^{* *}$ & $0,347^{* *}$ & NS & NS & NS & NS \\
\hline Côté tendu & NS & $-0,421^{* *}$ & $-0,372^{* *}$ & $0,520^{* *}$ & NS & NS & NS & NS \\
\hline
\end{tabular}

On observe des liaisons négatives avec les diamètres, et positives avec le retrait axial, ce qui tend à montrer que, lorsque l'angle d'inclinaison des tiges augmente, les contraintes s'aggravent, et le bois de tension s'étend.

La surprise vient du fait que le côté comprimé manifeste les mêmes tendances; certes pour les 3 paramètres les plus importants (diamètre tangentiel, somme des diamètres et retrait axial), les coefficients de corrélation sont nettement plus élevés du côté tendu, et ceci rejoint l'hypothèse émise plus haut pour expliquer que la variabilité de ces caractères y soit plus grande, mais on ne comprend pas pourquoi celle observée du côté comprimé est plus faible que pour les échantillons d'arbres droits, alors que l'influence prouvée de l'inclinaison vient y apporter une source de variation supplémentaire. 


\section{4. - Conclusion}

L'importance des effets de diverses origines observés, le fait que certaines différences statistiquement significatives sur les diamètres ne portent pas sur plus de 1,5 ou 2 microns, témoignent de la sensibilité et de la précision de la méthode de mesure utilisée.

Pour le reste, et bien que cela naait pas été le but premier de lexpérience, il convient de mentionner l'influence bénéfique des éclaircies sur le retrait radial, mais, en revanche, défavorable sur le retrait tangentiel et sur lanisotropie; cependant deux remarques suimposent : tout daabord, des résulhats en apparence contraires ont été obtenus dans le domaine voisin des relations entre rétractibilité et ampleur du houppier (Polge, 1973); par ailleurs, le retrait du bois est tributaire des contraintes qui se produisent pendant le séchage, qui varient en fonction de la forme et des dimensions des pièces de bois concernées; il diffère donc aussi bien entre une carotte de $5 \mathrm{~mm}$ de diamètre et un cube normalisé, quientre celui-ci et une planche par exemple. Dautres études seront donc nécessaires pour bien connaître les effets de la sylviculture sur la rétractibilité du hêtre, et aussi pour mieux établir, à la fois par des calculs théoriques et par des recherches appliquées, les relations entre retraits sur prélèvements non-destructifs effectués à la tarière et bois à dimensions d'emploi.

En matière de contraintes de croissance en revanche, les résultats du présent travail vont dans le même sens que ceux d'études antérieures ayant abordé des domaines voisins : ainsi, dans le compte rendu de l'expérience sur 86 hêtres disséminés dans un grand nombre de forêts françaises à laquelle il a été fait référence plus haut (Gueneau \& Saurat, 1974), 12 arbres de taillis-sous-futaie sur 36 dépassaient un certain seuil de contraintes (appréciées par la surface des courbes polaires des microdéformations), alors que cette proportion s'élevait à 35 sur 50 pour les arbres de futaie. De même, dans une comparaison faite à même altitude entre hêtres d'alignement et hêtres de forêt dans le département de la Corrèze (Al_ligreT, 1976), le niveau des contraintes des premiers ćtait apparu comme significativement inférieur à celui des seconds, au seuil de 1 p. 100. Lensemble des observations faites jusqu ici sur le hêtre montre donc que, si l'on veut éviter de produire du bois déprécié à l'excès par la présence de contraintes élevées, il faut s'efforcer d'obtenir des arbres à houppiers bien développés et pratiquer des éclaircies vigoureuses. Ce résultat est dautant plus important pour les sylviculteurs que lon aboutit à des conclusions semblables Iorsque lon a en vue la production de hêtre à faible dureté et à faible densité (Polge, 1973, op. cit.: Keiler, le Tacon, Timbal, 1976).

La présente étude laisse sans réponse un certain nombre de questions, notanment sur lampleur des effets «colonne», et, à un moindre degré, «ligne»; elle devra être reprise lorsque seront disponibles les données dendrométriques relative à ce dispositif, et lorsquil sera par suite possible de voir dans quelle mesure elles sont soumises aux mêmes sources de variation, et danalyser les liaisons entre elles. et les critères de qualité du bois.

Reçu pour publication en décembre 1980. 


\title{
Remerciements
}

Que soient vivement remerciés ici :

- M. Jean-René Perrin, qui a conçu et construit le capteur de mesure des diamètres, sans lequel ce travail naurait pas été possible:

- La Station de Sylviculture de Production, qui a bien voulu autoriser les prélèvements nécessaires dans le dispositif expérimental de Sonilly réalisé par elle;

- Les Techniciens de la Station de Recherches sur la Qualité des Bois qui ont procédé aux opérations de terrain et aux mesures de laboratoire;



\author{
Summary \\ Influence of the thinning regime of the growth stresses in beech
}

2 increment core have been taken in 320 beech trees to appreciate the growth stress level (when the fresh axial and tangential core diameters, measured with an accuracy of \pm 1 micron, are small, the stresses are high). The experimental design was a latin square with 4 treatments: ultra-heavy, heavy and low thinning regime and control.

On straight stems, the diameters regularly increase with the rate of thinning. On leaning stems, the 2 diameters are lower and the 3 shrinkages (axial, radial and tangential) higher, in the upper face than in the opposite one.

On the whole sampling the influence of the treatment is spectacular, e.g., for the sum of the axial and tangential diameters, that is probably the best indicator of the growth stresses, the 4 thinning regimes are quite separated from each other at the 1 p. 100 level, in the exact order of the intensity of thinning.

Negative correlations appear between fresh core dianeters and the axial shrinkage, confirming the association between high stresses and high proportion of tension wood. On leaning stems, diameters increase and the axial shrinkage decrease when the angle of the bole to the vertical decreases.

This experiment clearly show that heavy thinnings are to be made to reduce the growth stresses in beech stands.

\section{Références bibliographiques}

Alleiglet C., 1976. Le hêtre en Corrèze. D.D.A. de la Corrèze.

Boyd J.D., 1972. Tree Growth Stresses. Part. V : Evidence of an on Origin and Differentiation and Lignification. Wood Sci. and Technol., 6, 251-262.

Dinwoodif J.M.. 1966. Growth Stresses in Timber. A review of Literature. Forestry, XXXIX, 2, $162-170$.

FERRAND J.Ch., 1979. Appréciation des contraintes de croissance des arbres forestiers par mesure de carottes de sondage. 4' Congrès Français de Mécanique, 4-7 septembre 1979, Nancy.

Gueneau P., Kikata Y., 1973. Contraintes de croissance. Bois For. Trop., 149, mai-juin. 
Gueneau P., Saurat J., 1974. Contraintes de croissance. Rapport n" 1 : mesures en forêt. Cent. tech. Bois, convention 191126.

Keller R., Le Tacon F., Timbal J., 1976. La densité du bois de hêtre dans le Nord-Est de la France. Influence des caractéristiques du milieu et du type de sylviculture. $A n n$. Sci. for., 33, n" 1, 1-17.

Kubler H., 1959. Studien über Wachstumspannungen des Holzes. Erste Mitteilung : Die Ursache der Wachstumspannungen und die Spannungen quer zur Faserrichtung. Zweite Mitteilung : Die Spannungen in Faserrichtung. Hols als Roh-und Werkstoff, 17, $1-9,44-54$.

Lenz O., STrassler H.J., 1959. Contribution à l'étude de l'éclatement des billes de hêtre (Fagus silvatica L.). Mémoires de l'Institut Suisse de Recherches Forestières, Birmensdorf/ Zürich, 35, fasc. 5, 369-411.

Polge H., 1963. - Contribution à l'étude de la Qualité du bois des principales essences résineuses exotiques utilisées dans les reboisements français. Ann. Ec. natl. Eaux Forêts Stn Rech. et Exp. for., XX, fasc. 4.

Polg: H., 1973. Etat actuel des recherches sur la qualité du bois de hêtre. Bull. techn. O.N.F., 4, 13-22.

Polge H., 1979. Les contraintes de croissance sur le Hêtre. Résultats préliminaires sur l'influence de l'éclaircie. Document à distribution limitée Station de Recherches sur la Qualité des Bois du C.N.R.F., n" 1979/1.

Polge H., Thiercelin F., 1979. Growth stress appraisal through increment core measurements. Wood Sci., 12 (2), 86-92.

REINIKE: L.H., 1941. A new increment core instrument and coring wrinkles. J. For., 39 (3), 304-309.

Station de Sylviculture et de Production, C.N.R.F., 1970. Le «carré latin» de Souilly, dispositif expérimental d'éclaircic du hêtre. Document à distribution limitée, juin 1970.

Trenard Y., Gueneau P.. 1975. Relations entre contraintes de croissance longitudinales et bois de tension dans le hêtre. Holzforschung, 29, Heft 6, 217-223.

Trada Library Bibliography, 1978. Growth stresses. Timber Research and Development Association, Buckinghamshire. 